\title{
Evolução dos conhecimentos, atitudes e práticas relativas ao HIV/Aids em uma população de favela do Rio de J aneiro
}

\author{
Trends in HIV/Aids-related knowledge, attitudes, \\ and practices in a Rio de J aneiro slum population
}

João Claudio Lara Fernandes 1

1 Posto de Saúde da
Associação dos Moradores
e Amigos do Bairro Barcellos.
Travessa Flores 4,
Rio de Janeiro, RJ
$22451-000$, Brasil.
A bstract The study aims to eval uate the present stage of knowledge, attitudes, and behavior related to HIV/AIDS in the population of Rio de Janei ro's Rocinha slum, the target of a control program over the last 6 years. We interviewed two hundred and ten people of both sexes, ranging from 13 to 49 years of age, and the resul ts were compared with those of a study conducted in 1990 with the same methodol ogy and sample size. The analysis showed an association between single males and more preventive behavior. Misconceptions about the role of mosquito bites and blood donation in the transmission of HIV persist, almost in the same proportion. Comparing the two samples, there was a significant increase in the role of HIVIAIDS education provided by schools, and the study also identified an increase in the rates and regularity of condom use. More efforts should be made to reduce misconceptions about HIV transmission and the vulnerability of couples. The study al so highlights the need for more consistent policies rel ated to condom distribution to the general population.

Key words HIV; Acquired Imunodeficiency Syndrome; Knowledge, Attitudes, Practice; Condoms

Resumo O estudo visa acessar o estágio atual de conheci mentos, atitudes e comportamentos re lativos ao HIV/Aids na população da favela da Rocinha, a qual vem sendo abrangi da por um programa de controle há 6 anos. Foram entrevistados 210 moradores com idade entre 13 e 49 anos, de ambos os sexos, comparando-se os resul tados com os de um outro estudo real izado em 1990, com a mesma metodologia e tamanho amostral. Observou-se uma associação significativa entre o fato de ser sol tei ro e do sexo mascul ino, com um mai or índice de adoção de comportamentos preventivos. Persistem concepções incorretas quanto ao papel do mosquito e do ato de doar sangue, na transmissão do vírus. Comparando-se os dois estudos realizados, houve um aumento significativo da importância da escola na transmi ssão de informações sobre o HIV/Aids, e um aumento no uso de preservativos. São sugeridos novos esforço, a fim de mel horar a informação sobre as formas de transmissão do vírus, bem como de apoio à reflexão e deci são comportamental dos casais. É sal ientada a necessi dade de estabelecimento de políticas consistentes de distribuição de preservativos para a população em geral.

Palavras-chave HIV; Síndrome de Imunodeficiência Adquirida; Conhecimentos, Atitudes e Prática; Preservativos 


\section{Introdução}

O Programa Comunitário de Controle da Aids (PCCA) foi iniciado em 1990, e tem como objetivo o desenvolvimento de ações de assistência e prevenção de DST/Aids junto aos moradores da comunidade da Rocinha. O programa é coordenado pelo Posto de Saúde da Associação de Moradores e Amigos do Bairro Barcellos (AMABB) e, a partir de 1996, passou a contar com financiamento do Ministério da Saúde - Programa Nacional de DST/Aids.

Para levantar informações preliminares sobre os conhecimentos, percepções e atitudes relativas ao HIV/Aids na comunidade, foi realizado um primeiro inquérito em 1990 (Fernandes et al., 1992). Passados seis anos de atividades, realizou-se um segundo estudo, para avaliar as evoluções ocorridas neste período e identificar questões a serem abordadas no trabalho educativo.

\section{Características da população}

A favela da Rocinha situa-se na zona sul do Rio de Janeiro e possui uma população estimada em 150.000 habitantes, boa parte da qual é constituída por imigrantes nordestinos. A atividade econômica predominante relaciona-se ao setor de prestação de serviços. Existe um alto índice de iniciação sexual e gravidez precoces, bem como de atividades de comércio sexual, além de uma forte presença do tráfico de drogas.

Os primeiros casos conhecidos de Aids na comunidade datam de 1986, e até junho de 1995 haviam sido registrados 79 moradores com HIV/ Aids. Destes, 27 eram mulheres e 52 homens, o que dá uma relação de 2 homens para cada mulher infectada. As faixas etárias mais acometidas estão situadas entre 20 e 40 anos, e há 1 ocorrência de transmissão vertical. $\mathrm{Na}$ grande maioria dos casos a via de contaminação foi o relacionamento sexual.

\section{Metodologia}

Trata-se de um estudo transversal, abrangendo a população compreendida entre 13 e 49 anos, de ambos os sexos, moradores da Rocinha. Para o procedimento amostral foi feita uma adaptação do método proposto por Frerichs \& Tar Tar (1989), que consistiu na divisão da área da favela em 30 setores demograficamente homogêneos, identificando-se, em cada um destes, 5 pontos de referência. Em cada setor foi sortea- do um ponto, a partir do qual o entrevistador gira uma seta, que fornece a direção a ser percorrida. Nesta direção, os sete primeiros residentes identificados são entrevistados, perfazendo-se um total de 210 entrevistas. Este procedimento, já utilizado em outros estudos (Fernandes et al., 1992; SES-RJ/ Abia, 1994), busca superar as dificuldades relacionadas ao correto mapeamento ou registro censitário deste tipo de comunidade, as quais inviabilizam a opção pelo sorteio direto de domicílios ou moradores.

Para a obtenção dos dados foi confeccionado um questionário com 49 perguntas com respostas abertas e fechadas. As respostas não previstas no instrumento foram codificadas posteriormente. Também foi elaborada uma ficha de domicílio, para anotação de dados dos entrevistados e recusas.

Para a execução da pesquisa foram selecionados 5 entrevistadores, membros da comunidade, que receberam um treinamento de 10 horas. O trabal ho de campo foi realizado nos dias 20 e 21 de julho de 1996.

A análise dos dados foi feita com o auxílio do programa Epilnfo 6.0, e consistiu na confecção de tabelas de freqüência e algumas estratificações que se mostraram consistentes, mesmo considerando-se o tamanho da amostra.

\section{Resultados}

A população entrevistada caracterizou-se por um predomínio de mulheres $(70,5 \%)$ e pessoas casadas (56\%), com uma idade média de 29 anos. $\mathrm{O}$ grupo de mulheres casadas foi significativamente superior aos de homens solteiros, casados e mulheres solteiras $(p<0,03)$. Em relação à naturalidade, $45 \%$ eram nascidos no Rio de Janeiro, enquanto $25 \%$ eram oriundos do Ceará e $11,5 \%$ da Paraíba. Noventa e cinco por cento da amostra haviam freqüentado a escola, tendo $38 \%$ desta alcançado da $3 a$ à $5 a$ a série do lo grau.

As maiores fontes de informação sobre HIV/Aids referidas pelos entrevistados foram a televisão (56\%), a escola (13,5\%) e pessoas da própria comunidade $(6,8 \%)$. A Tabela 1 resume os principais dados obtidos em relação aos conhecimentos sobre a infecção.

Em relação ao campo das percepções, 83\% dos entrevistados percebiam a Aids como uma ameaça à comunidade, e 3/4 da amostra sabiam de casos ocorridos na Rocinha. Cinqüenta e sete por cento das pessoas já haviam conhecido pessoal mente al guém infectado. Por outro lado, 1/ 4 dos respondentes duvidava da 
eficiência da camisinha como meio de proteção.

Cerca de $11 \%$ da amostra não haviam iniciado sua vida sexual. A Tabela 2 mostra as respostas obtidas em relação ao comportamento sexual.

Trinta por cento da amostra compravam camisinhas, principalmente em farmácias, e 9,2\% as obtinham através de Educadores Comunitários do PCCA.

Comparando-se pessoas solteiras e casadas (ou que moram com seu parceiro), observouse, no primeiro grupo, uma associação estatisticamente significativa $(p<0,01)$ em relação à referência à mudança de comportamento, ao uso de preservativo, ao uso regular de preservativos, ao uso deste meio na última relação sexual, e à intenção de uso, em caso de distribuição gratuita, como mostra a Figura 1.

Na medida em que o grupo de pessoas casadas apresentou um forte predomínio de muIheres, não foi surpresa observar uma associação entre o sexo feminino e uma menor referência à mudança de comportamento sexual ( $p<0,0001)$, ao uso de preservativo $(p=0,03)$, e ao uso regular deste meio $(p=0,03)$.

\section{Comentários}

Nos primeiros anos da epidemia do HIV, quando ainda eram grandes as lacunas de conhecimento sobre os comportamentos sociais relacionados ao novo fenômeno, os inquéritos de tipo KAPB (Knowledges, Attitudes, Practices and Beli efs) foram bastante utilizados, na medida em que permitiam o acesso rápido a determinadas características comportamentais de uma população. Na atual década, observa-se uma tendência à valorização de estudos de natureza mais qualitativa, que podem clarear com mais eficiência as complexas inter-relações da epidemia com as questões da sexualidade, cultura e sociedade (ARHNWGSB, 1995; Parker et al., 1996). Apesar disso, consideramos que aquela metodologia segue tendo sua utilidade, particularmente na avaliação de atividades educativas e na identificação, grosso modo, de estágios de evolução de uma população no seu processo de relacionamento com a epidemia. De todo modo, em função de suas deficiências intrínsecas, esta metodologia deve, sempre que possível, ser complementada por estudos de natureza qualitativa, mais indicados para a elaboração de estratégias de prevenção. Considerando esses aspectos, a intenção deste estudo foi a de observar, ainda que superficialmente, como evoluíram certos tipos de informação
Tabela1

\begin{tabular}{lc}
\hline Conhecimentos relativos ao HIV/Aids & $\%$ \\
\hline Inexistência de vacinas eficazes & 74,4 \\
Inexistência de remédios capazes de curar a Aids & 74,9 \\
Uma pessoa de boa aparência pode ter o vírus & 90,8 \\
Um homem infectado pode transmitir para a mulher, na relação sexual & 96,1 \\
Uma mulher infectada pode transmitir para o homem, na relação sexual & 96,6 \\
Um homem infectado pode transmitir para outro homem, na relação sexual & 95,7 \\
A transfusão de sangue pode provocar a contaminação de uma pessoa & 97,1 \\
A doação de sangue pode contaminar & 56,0 \\
O beijo na boca pode transmitir o vírus & 20,3 \\
A picada de mosquito pode transmitir o vírus & 41,1 \\
A mãe pode transmitir ao filho, durante a gravidez & 91,8 \\
\hline
\end{tabular}

Tabela 2

\begin{tabular}{lc}
\hline Comportamentos relativos ao HIV/Aids* & $\%$ \\
\hline Mudou o comportamento sexual & 36,7 \\
Já usou preservativo & 57,0 \\
Usa preservativo & 41,1 \\
Usa o preservativo regularmente & 16,4 \\
Usou preservativo na última relação sexual & 15,9 \\
Usaria preservativo, caso os recebesse gratuitamente & 58,5
\end{tabular}

* Excluídas as pessoas sem iniciação sexual.

(ou desinformação) e comportamentos relativos ao HIV/ Aids em uma população determinada, que vem sendo abrangida por um programa de controle há seis anos.

Cabe ressaltar que não houve, neste caso específico, uma intenção avaliativa em relação aos resultados do PCCA, em virtude do longo tempo transcorrido entre os dois inquéritos realizados ( 6 anos), e também pelos vieses intrínsecos a este desenho de avaliação. De fato, a aferição de resultados de um projeto de prevenção baseada em estudos do tipo antes-depois, não leva em conta os numerosos fatores externos a ele, capazes de interferir no comportamento de uma população (Coyle et al., 1991). Este aspecto é particularmente importante no que se refere ao tema do HIV/Aids, continuamente abordado na mídia em geral, e que tem se caracterizado pela incessante descoberta de novos ângulos, que vão alterando, certamente, o imaginário social sobre a infecção, tais como potenciais novas formas de contaminação, resultados de tratamentos, etc. Neste ponto, deve-se assinalar que nosso estudo foi realizado pouco depois da IX Conferência Internacional sobre Aids, durante a qual foram amplamente divulgados resultados promissores do coquetel anti-retroviral. Estas notícias 
Figura 1

Comportamentos relativos ao HIV/Aids segundo a moradia ou não com parceiro/a.

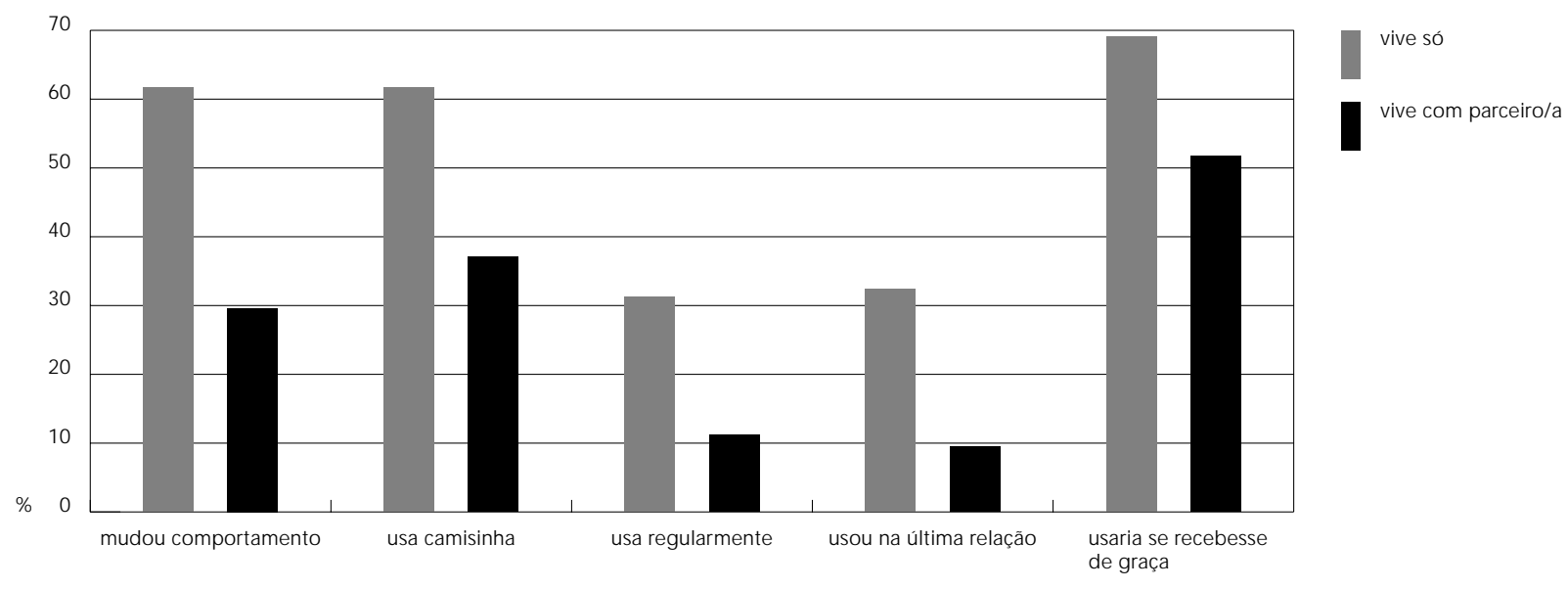

podem ter influenciado algumas das respostas do inquérito, como as referentes à curabilidade da Aids. Por outro lado, o inquérito realizado anteriormente, com a mesma metodologia e tamanho amostral, é uma referência óbvia para a avaliação das mudanças e permanências observadas naquela população, motivo pelo qual nos referiremos a ele sempre que considerarmos adequado. Nestes casos, para uma meIhor avaliação dos efeitos das possíveis flutuações nas características das duas amostras, utilizaremos o teste do Qui-quadrado, para mensurar o grau de significância estatística das diferenças observadas.

Em relação às respostas obtidas na investigação, um primeiro aspecto a ser considerado refere-se ao aparecimento da escola como a segunda fonte de informação mais mencionada pelos entrevistados, lugar que era ocupado, no inquérito anterior, pelo rádio. Naquele estudo, a escola ocupava um humilde 60 lugar entre as fontes relatadas. A diferença entre os dois inquéritos mostrou-se bastante significativa, $\operatorname{com} p=0,00001$. Esta mudança indica possivelmente um maior engajamento das instituições de ensino na conscientização dos jovens em relação ao HIV, o que é um dado alvissareiro, e demonstra a importância deste setor entre as diversas áreas de atuação preventiva.

Um outro aspecto que ressalta da análise é que, apesar de todos estes anos e de todo o esforço informativo realizado, persiste a mesma proporção de respondentes que qualificam o mosquito como vetor da infecção. No inquérito realizado em 1990, 45,2\% da amostra com- partilhavam desta opinião (Fernandes et al., 1992), referida por $41,1 \%$ dos respondentes no atual estudo. Resultados semelhantes têm sido encontrados em diversos trabal hos: Goldstein (1995) cita uma pesquisa do DataFolha, de 1991, realizada em quatro capitais brasileiras, na qual $52 \%$ dos entrevistados faziam referência à picada de mosquito como potencial fator de contaminação. Uma pesquisa realizada nos Estados Unidos revela uma proporção de 57\% de respondentes com a mesma opinião (Médicine et Hygiene, 1991), e uma outra investigação, dirigida a uma população universitária da Tailândia, refere $42 \%$ deste tipo de resposta (Jayasuriya \& Phlainoi, 1992). Ainda que, em nosso estudo, não se tenha observado diferença estatística entre aqueles que partilham deste ponto de vista e os restantes no que se refere à credibilidade no preservativo e ao uso deste meio de prevenção, é bastante plausível considerar que este viés na informação sobre o HIV pode levar à perda do sentido de utilidade da adoção de comportamentos preventivos, conduzindo a uma percepção fatalista e paralisante do problema.

Um outro viés que parece persistir na informação sobre o HIV relaciona-se à doação de sangue, percebida como fator de transmissão por $61 \%$ dos entrevistados, no primeiro inquérito, e por $56 \%$, no presente estudo $(p=0,3)$. Um estudo realizado com 1200 moradores de 4 favelas do Rio de Janeiro, em 1993, encontrou este tipo de resposta em $52 \%$ dos entrevistados (SES/ Abia, 1994). Este dado está provavelmente relacionado à confusão entre o ato de doar e 
de receber sangue, e indica que maiores esforços devem ser feitos para a compreensão dos fatores subjacentes a este tipo de percepção, bem como para uma abordagem mais clara desta questão na mídia.

Observou-se uma associação sistematicamente consistente entre o fato de ser solteiro (morar sem um parceiro/a) e a adoção de hábitos mais seguros em relação à infecção, como foi mostrado na Figura 1. Resultado semelhante foi obtido no estudo, já referido, em 4 outras favelas do Rio de Janeiro, que revelou uma associação significativa $(p<0,05)$ entre o fato de ser solteiro (isto é, não morar com parceiro) e uma maior referência ao uso de preservativo, ao uso regular deste meio, e à intenção de uso, caso o recebesse gratuitamente. Embora estes dados em si, do ponto de vista do senso comum, não sejam uma surpresa, não deixam de apontar um problema no que se refere às pessoas com relacionamentos estáveis, ao menos no tipo de população estudada, se considerarmos as tendências epidemiológicas da infecção pelo HIV. Segundo o Ministério da Saúde, no período de 1980 a 1988, 53\% dos casos de Aids estavam relacionados a práticas homo e bissexuais, enquanto que apenas 3,1\% eram atribuídos à relação heterossexual. Já em 1995, esta última proporção sobe para $29,1 \%$, contra $23 \%$ de registros vinculados aos comportamentos homo-bissexuais (Dados, 1996). Também já é bastante conhecido o aumento relativo (e absoluto) da infecção em mulheres, que passa de 1 caso para cada 9 masculinos, em 1989, para 1 em 3 casos masculinos, em 1995. Um estudo realizado pela Secretaria de Estado de Saúde do Rio de Janeiro revelou que 37,5\% das mulheres portadoras de Aids são donas de casa (SES-RJ, 1996). Estes dados indicam o aumento da vulnerabilidade da população em geral, aí incluídas as pessoas com relacionamentos estáveis, e projetam especificamente um problema de altíssima transcendência, que é a perspectiva do aumento da orfandade relacionada ao HIV/ Aids. É fundamental, portanto, promover a conscientização dos casais, a fim de assumirem comportamentos mais seguros em relação à infecção. Não estamos nos referindo, aqui, a uma intenção aprioristica de disseminação do uso da camisinha pelas pessoas com relacionamentos estáveis, o que nos parece um tanto surrealista, mas à necessidade de fortalecimento da reflexão conjugal sobre a questão do HIV, de modo a propiciar processos autênticos de decisão comportamental, por parte de cada casal (Fernandes, 1994).

Um outro aspecto apontado neste estudo refere-se ao aumento da utilização do preser-
Figura 2

Evolução no consumo de preservativos.

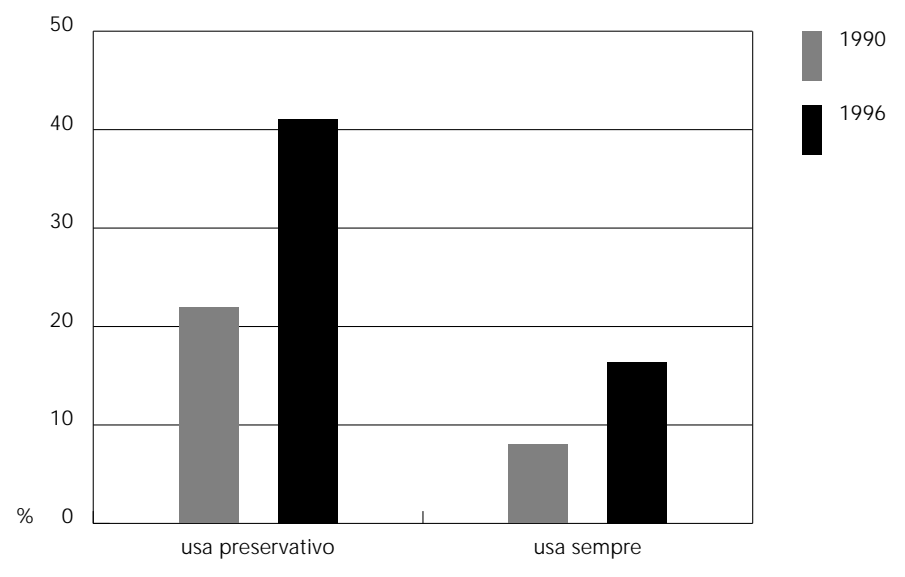

vativo, em relação ao inquérito realizado anteriormente. Como indica a Figura 2, a proporção de entrevistados que referiram utilizar este meio era de $21,9 \%$ em 1990 , contra $41,1 \%$ em 1996 ( $p \varangle 0,0001$ ). Também o uso regular da camisinha, encontrado em $8 \%$ da primeira amostra, dobrou no presente estudo, passando a $16,4 \%$ dos respondentes ( $p=0,01)$. Estes dados indicam uma evolução favorável da adoção de comportamentos preventivos naquela comunidade. Ainda que não se possa atribuir este fato ao desempenho do programa realizado, cabe notar que quase $10 \%$ da amostra recebiam camisinhas de Educadores Comunitários, o que indica, ao menos, uma participação importante do programa no processo de mudança comportamental. É difícil a comparação da freqüência atual de uso de preservativos na Rocinha com a de outros grupos sociais. A maior parte das pesquisas realizadas com a intenção de estimar as proporções de utilização deste método abrangem, geralmente, populações bastante diferenciadas, em função de comportamentos específicos (trabal hadores sexuais, UDIs, homossexuais), ou por faixas etárias (adolescentes) e gênero (mulheres), ou, enfim, por padrões sócio-econômico-culturais, como ocorre nos estudos realizados em outros continentes. Por outro lado, o inquérito realizado em 4 favelas, já referido anteriormente, revelou uma média de $28,4 \%$ de utilização da camisinha, com $12,6 \%$ de uso regular deste meio (SES-RJ/ Abia, 1994).

De qualquer modo, a discussão sobre os significados da freqüência de uso de camisi- 
nhas encontrada no estudo é certamente menos importante do que outra, relacionada à afirmação, obtida no inquérito, por parte de $58 \%$ dos entrevistados, de que usariam o preservativo, caso o recebessem gratuitamente. Esta mesma pergunta foi colocada na pesquisa realizada em 4 outras favelas do Rio, obtendose, em média, os mesmos $58 \%$ de respostas afirmativas (SES-RJ/ Abia, 1994). Estes dados, mesmo considerando-se a superficialidade inerente ao método utilizado, levantam questões que precisamos aprofundar.

Em primeiro lugar, existe hoje um amplo reconhecimento de uma tendência epidemiológica crescente da infecção pelo HIV nas camadas mais pobres da população - ao mesmo tempo em que da hetero-sexualização. Em um estudo realizado pela SES-RJ em 1996, constatou-se que a proporção de casos de Aids com nível de escolaridade universitário evoluiu de $50,7 \%$ (1980-89) para 21,7\% (1990-96), enquanto que a freqüência de pacientes com instrução até o 1 o grau aumentou de $18,8 \%$ dos casos, no período de 1980-89, para 51,2\%, em 199096 (Figura 3). Este dado não chega a surpreender, considerando-se a história da maioria das doenças infecto-contagiosas em nosso país, e considerando-se, ainda, a falta de informação e de acesso aos meios de prevenção, diagnóstico e tratamento, fatores que aumentam a vulnerabilidade dos segmentos mais pobres da população no tocante à questão do HIV/ Aids.

Um panorama diferente é exibido pelos países desenvolvidos, onde o acesso ao preservati-

\section{Figura 3}

Distribuição dos casos novos de Aids no Rio de J aneiro segundo a escolaridade (SES-RJ, 1996).

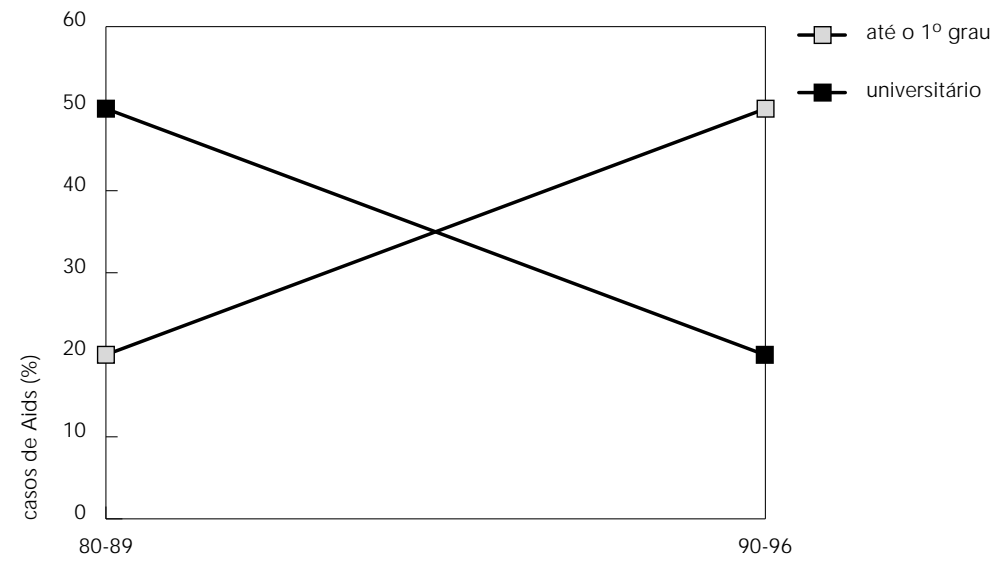

vo é bastante facilitado, tornando central, nestes, a questão de aprofundar os meios de persuasão para sua mais ampla utilização. Em outras palavras, o problema da mudança comportamental é o principal desafio que estes países enfrentam na luta contra a epidemia. Os numerosos estudos, pesquisas e análises elaborados nestes países, com este objetivo, claramente influenciam as análises e ações elaboradas nos países em desenvolvimento, como o nosso, e podem levar a uma interpretação restrita da nossa realidade. Considerando-se os dados apresentados no presente estudo, eas tendências epidemiológicas acima referidas, podemos levantar a hipótese de que a questão central para o controle do HIV/Aids em nosso país é a facilitação do acesso da população aos meios de prevenção. Cabe lembrar, aqui, que atualmente uma caixa com três preservativos custa, em média, o equivalente a 4 litros de leite e, deste modo, é bastante plausível supor que um contigente da população utilizaria este método - ainda que por intenções contraceptivas - de bom grado, caso pudesse adquiri-lo.

Não estamos, com isto, menosprezando o papel fundamental das pesquisas relacionadas à mudança comportamental, das quais este estudo é um humilde exemplo, e muito menos a relevância das ações educativas relacionadas à prevenção do HIV/Aids. Queremos, de fato, ampliar o foco destas ações, e chamar atenção para o aspecto da facilitação do acesso ao preservativo, que coloca na pauta das discussões relacionadas ao controle da epidemia a necessidade urgente de elaboração de uma política efetiva de distribuição deste meio, sem a qual corre-se o risco de tornar um tanto bizantina boa parte das discussões relacionadas à mudança comportamental e de tornar inútil boa parte do esforço educativo realizado, além, obviamente, do desperdício de recursos resultante deste fato.

\section{Conclusão}

Esta pesquisa teve como objetivo central a avaliação do estágio atual de conhecimentos e comportamentos da população da Rocinha em relação ao HIV/ Aids. Com base na comparação com o estudo realizado 6 anos antes, é possível delinear algumas tendências:

- Uma maior participação da escola enquanto fonte de informação sobre HIV/Aids.

- A permanência de certos vieses de conhecimento, como o papel do mosquito e da doação de sangue na disseminação do vírus.

- A duplicação da freqüência de uso do preservativo na comunidade. 
Além disso, e mesmo considerando-se que o estudo abrange uma população específica, geograficamente delimitada, a comparação com outras pesquisas, particularmente aquelas dirigidas ao mesmo segmento social, autoriza algumas extrapolações, ainda que em um nível indicativo. A partir disto, podemos resumir algumas recomendações mais gerais a serem consideradas:

- Novos esforços são necessários, a fim de esclarecer a população sobre as relações entre o ato de doar e receber sangue e a contaminação

\section{Agradecimentos}

O autor agradece ao Dr. Evandro Coutinho pelas sugestões e críticas ao texto.

\section{Referências}

ARHNWGSB (Aids and Reproductive Health Network's Working Group on Sexual Behavior), 1995. Gender, Sexuality and Health: Building a New Agenda for Sexuality Research in Response to Aids and Reproductive Health. Rio de Janeiro: ARHNWGSB.

COYLE, S. L.; BORUCH, R. F. \& TURNER, C. F., 1991. Evaluating Aids Prevention Programs. Washington, D.C.: National Academy Press.

DADOS, 1996. Aids: Uma Epidemia em Mutação. Rio de Janeiro. RADIS/ENSP.

FERNANDES, J. C. L.; COUTINHO, E. S. F. \& MATIDA, A. H., 1992. Conhecimentos e atitudes relativas à SIDA/Aids em uma população de favela do Rio de Janeiro. Cadernos de Saúde Pública, 8:176-182.

FERNANDES, J. C. L., 1994. Práticas educativas para a prevenção do HIV/Aids: aspectos conceituais. Cadernos de Saúde Pública, 10:171-180.

FRERICHS, R. R. \& TAR TAR, K., 1989. Computer-assisted rapid surveys in developing countries. Public Health Reports, 104:14-23. pelo HIV, e também no que se refere à picada do mosquito enquanto fator de infecção.

- É necessário incentivar a discussão sobre o HIV na população em geral, particularmente os casais, de modo a fortalecer o processo de decisão comportamental e reduzir a vulnerabilidade à infecção.

- Finalmente, urge efetuar as pressões políticas necessárias para o estabelecimento de uma política consistente de acesso da população em geral ao preservativo.
GOLDSTEIN, D., 1995. The Cultural, Class and Gender Politics of a Modern Disease: Women and Aids in Brazil. Washington, D.C.: International Center for Research on Women.

JAYASURIYA, D. C. \& PHLAINOI, N., 1992. Knowledge of HIV/ Aids safety practices among Thai undergraduates. Journal of Primary Health Care and Development, 5:1-10.

MÉDICINE ET HYGIĖNE, 1991. Encore bien loin de la victoire sur le SIDA. Médicine et Hygiene, 49:3346.

PARKER, R.; BARBOSA, R. \& FAJARDO, E., 1996. Novas tendências da pesquisa em gênero, sexualidade e saúde. Sexualidade: Gênero eSociedade, 5:1.

SES-RJ/ ABIA (Secretaria de Estado de Saúde do Rio de Janeiro/Associação Brasileira Interdisciplinar de Aids), 1994. Relatório do Programa Regionalizado de Controle da Aids. Rio de Janeiro: Abia.

SES-RJ (Secretaria de Estado de Saúde do Rio de Janeiro), 1996. Dados sobre Aids no Estado. Rio de Janeiro. Divisão de DST/Aids. Secretaria de Estado de Saúde do Rio de Janeiro. 